\title{
Pestizide können Demenz verursachen
}

\author{
Wer beruflich intensiv mit Pestiziden \\ in Kontakt kommt, hat ein signi- \\ fikant erhöhtes Risiko, dement zu \\ werden. Dies ergab die PHYTONER- \\ Studie aus Frankreich.
}

- In der Phytoner-Studie untersuchten Wissenschaftler in Bordeaux (Frankreich) die Langzeitauswirkung der Pestizidexposition auf die neurologischen Verhaltensleistungen bei französischen Weinbergarbeitern.

929 Arbeiter im Gebiet von Bordeaux waren beteiligt. Sie wurden kontaktiert, füllten einen Fragebogen aus und führten über einen Zeitraum von sechs Jahren neun neurologische Verhaltenstests durch. Die Probanden wurden hinsichtlich Pestizidexposition über mindestens 20 Jahre in drei Gruppen klassifiziert: direkt (Mischen und Anwenden der Pestizide, Reinigen und Reparieren der Sprühausrüstung), indirekt (Kontakt mit behandelten Pflanzen, Arbeiten an Gebäuden, Büros und Kellern) und nicht exponiert. Der Ausbildungsgrad, das Alter, das Geschlecht, der Alkoholkonsum, das Rauchen, die Einnahme psychotroper Medikamente und depressive Symptome wurden in der Analyse berücksichtigt.

614 Personen waren zur Auswertung geeignet. Das Risiko, in den Tests niedrige Leistungen zu erreichen, war bei den exponierten Arbeitern mit OR von 1,35-5,60 signifikant erhöht. Das Risiko, einen um zwei Punkte niedrigeren Wert bei den Tests zu erreichen, war bei den exponierten Personen mit 2,15; 95\%-KI=1,18-3,94 mehr als doppelt so hoch. Auch war die Progredienz der Demenzsymptomatik bei den exponierten Personen besonders ausgeprägt.

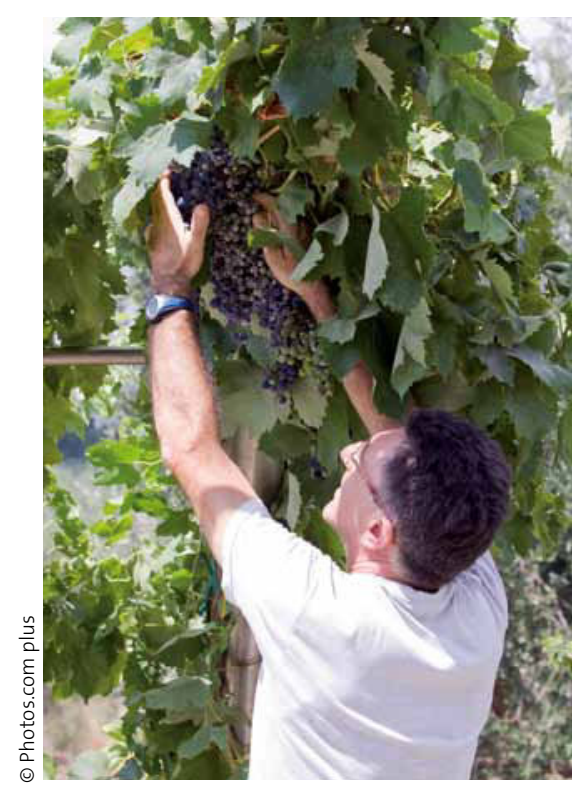

Arbeiter im Weinanbau sind einer hohen Pestizidbelastung ausgesetzt.

Kommentar

Dies ist die erste prospektive Studie, die Daten zur Korrelation von Pestizidexposition und Demenzentwicklung liefert. Die Resultate suggerieren chronische Langzeiteffekte der Pestizide und eine Zunahme des Demenzrisikos.

\section{K. MALBERG =}

\footnotetext{
- I. Baldi et al.

(Laboratoire Santé Travail Environnement, EA 3672, Institut de Santé Publique d'Epidémiologie et de Développement, 146 rue Léo Saignat, 33076 Bordeaux Cedex, France; isabelle.baldi@isped.u-bordeaux2.fr) Neurobehavioral effects of long-term exposure to pesticides: results from the 4year follow-up of the PHYTONER Study. Occupational and Environmental Medicine, 2010; DOI: 10.1136/oem.2009.047811
} 